\title{
A cognitive training intervention increases resting cerebral blood flow in healthy older adults
}

\author{
Jennifer L. Mozolic ${ }^{1,2 *}$, Satoru Hayasaka ${ }^{3}$ and Paul J. Laurienti ${ }^{2}$ \\ 1 Neuroscience Program, Wake Forest University School of Medicine, Winston-Salem, NC, USA \\ 2 Department of Radiology, Wake Forest University School of Medicine, Winston-Salem, NC, USA \\ ${ }^{3}$ Department of Biostatistics, Wake Forest University School of Medicine, Winston-Salem, NC, USA
}

\section{Edited by:}

Marty G. Woldorff, Duke University,

USA

\section{Reviewed by:}

Heleen A. Slagter,

University of Wisconsin, USA

Clifford D. Saron,

University of California at Davis, USA

*Correspondence:

Jennifer L. Mozolic, Department of Radiology, Wake Forest University

School of Medicine, Medical Center

Boulevard, Winston-Salem, NC 27157,

USA.

e-mail:jmozolic@warren-wilson.edu
Healthy aging is typically accompanied by some decline in cognitive performance, as well as by alterations in brain structure and function. Here we report the results of a randomized, controlled trial designed to determine the effects of a novel cognitive training program on resting cerebral blood flow (CBF) and gray matter (GM) volume in healthy older adults. Sixty-six healthy older adults participated in 8 weeks of either a training program targeting attention and distractibility or an educational control program. This training program produced significantly larger increases in resting CBF to the prefrontal cortex than the control program. Increases in blood flow were associated with reduced susceptibility to distraction after training, but not with alterations in $\mathrm{GM}$ volume. These data demonstrate that cognitive training can improve resting $\mathrm{CBF}$ in healthy older adults and that cerebral perfusion rates may be a more sensitive indicator of the benefits of cognitive training than volumetric analyses.

Keywords: aging, attention, distraction, perfusion, randomized controlled trial

\section{INTRODUCTION}

Changes that occur in the aging brain can produce alterations in a variety of sensory and cognitive functions. Even healthy older adults typically exhibit significant deficits in memory, attention, and other executive functions (Park et al., 2002; Verhaeghen and Cerella, 2002; Buckner, 2004; Rhodes, 2004; Isingrini et al., 2008). For example, in a large sample of adults age 20-92, Park et al. (2002) demonstrated age-related declines in across several cognitive domains, including speed of processing, working memory, and long-term memory. A number of studies also indicate that older adults process more extraneous sensory information and are more distractible than their young counterparts (Alain and Woods, 1999; Rowe et al., 2006; Yang and Hasher, 2007; Hugenschmidt et al., 2009).

The frontal cortex, which supports many of these higher-level cognitive functions, is known to be particularly susceptible to many age-related changes, including reductions in blood flow (Martin et al., 1991; Meltzer et al., 2000; Parkes et al., 2004; Bangen et al., 2007; Asllani et al., 2009; Bertsch et al., 2009), metabolic rates (Marchal et al., 1992; Bentourkia et al., 2000), white matter (WM) integrity (Salat et al., 2005; Hugenschmidt et al., 2008), tissue volume (Good et al., 2001; Resnick et al., 2003; Raz et al., 2004, 2005; Salat et al., 2009), and cortical thickness (Salat et al., 2004; Fjell et al., 2009). Although brain atrophy can artificially inflate the difference in cerebral blood flow (CBF) between younger and older adults, due to age-related decreases in the amount of gray matter (GM) relative to WM or cerebrospinal fluid present in a given voxel, a recent study by Asllani et al. (2009) reported that older adults have a global decrease in CBF of $\sim 15 \%$, even after correcting for these partial volume effects. In addition to confirming that there are global reductions in CBF, these experiments also demonstrated that tissue loss did not fully account for local age-related decreases in CBF in several brain areas, including the cingulate gyrus, the frontal cortex, and the hippocampus (Asllani et al., 2009). Interestingly, a longitudinal study of healthy older adults that examined $\mathrm{CBF}$ patterns during task and resting-state also demonstrated specific decreases in resting-state $\mathrm{CBF}$ in the parahippocampal and superior frontal gyri across an 8-year time span (Beason-Held et al., 2009). It is unknown whether improved blood flow to these brain regions could improve neuronal processing efficiency.

Despite these age-related alterations in brain structure and function, the aging brain does maintain some ability to adapt and change in response to environmental demands (Churchill et al., 2002; Kempermann et al., 2002; Kramer et al., 2004; Burke and Barnes, 2006; Mora et al., 2007; Jessberger and Gage, 2008). Several interventional studies have capitalized on this plasticity to improve cognitive function in older adults with training programs that target memory, attention, reasoning, and speed of processing (Kramer et al., 1995; Ball et al., 2002; Jennings et al., 2005; Mahncke et al., 2006; Erickson et al., 2007; Bherer et al., 2008; Buschkuehl et al., 2008; Dahlin et al., 2008a; Mozolic et al., 2009). In a large, randomized controlled trial known as the ACTIVE study, participants trained in memory, speed of processing, or reasoning showed significant improvements in the trained domains, and these improvements were maintained over a 5-year follow-up interval (Ball et al., 2002; Willis et al., 2006).

A number of other studies have used neuroimaging to identify modifications in brain structure and function that are associated with various training regimens (Draganski et al., 2004, 2006; Golestani and Zatorre, 2004; Olesen et al., 2004; May et al., 2007; Ilg et al., 2008; Hyde et al., 2009); however, fewer have investigated training-induced plasticity in the aging brain (Nyberg et al., 2003; Colcombe et al., 2004, 2006; Erickson et al., 2007; Pereira et al., 2007; Boyke et al., 2008; Dahlin et al., 2008b). Colcombe 
et al. (2004) demonstrated that cardiovascular fitness training in older adults improved performance and increased activity in the prefrontal and parietal cortex during performance on a task of executive function. Similar aerobic exercise interventions have also been shown to increase both GM volume in the anterior cingulate, right frontal, and left temporal cortices and WM volume in the anterior corpus callosum (Colcombe et al., 2006), and to increase resting cerebral blood volume in the hippocampus in healthy older adults (Pereira et al., 2007). Cognitive training interventions have also been shown to successfully alter task-related neural activity in older adults (Nyberg et al., 2003; Erickson et al., 2007; Dahlin et al., 2008b), yet little is known about alterations in resting brain activity or blood flow following cognitive training in older adults. Interestingly, in young adults, training in logical reasoning can improve performance on such tasks and increase resting $\mathrm{CBF}$ to the $\mathrm{PFC}$ and anterior cingulate cortex (ACC) (Mazoyer et al., 2009).

We have recently reported on the behavioral improvements that result from a multisensory attention training program in older adults (Mozolic et al., 2009); however it is unknown how these improvements may relate to underlying changes in brain function or structure. After completing an 8-week cognitive training program designed to decrease distractibility, older adults exhibited significantly greater reductions in interference from cross-modal distractors than controls. Improvements for participants in the treatment group also generalized to several other measures of executive function (Mozolic et al., 2009). In the present report, we investigate the effects of this training program on regional $\mathrm{CBF}$ during rest and GM volume. To our knowledge, this is the first reported investigation of cognitive training-induced changes in brain structure and resting CBF in healthy older adults. Specifically, we hypothesized that this training regimen, which required repeated practice at suppressing distracting stimuli during demanding cognitive tasks, would increase resting CBF in the prefrontal cortex (PFC), a brain area known to be involved in attention and inhibition (Corbetta and Shulman, 2002; Fassbender et al., 2004; Shomstein and Yantis, 2004; Weissman et al., 2004). Additionally, we expected that there would be an increase in GM volume in regions of the PFC supporting these executive functions trained during the intervention. Finally, we anticipated that changes in regional CBF and GM volume would be correlated with the behavioral improvements produced by this novel training program.

\section{MATERIALS AND METHODS}

This study used a single-blind, randomized controlled design to determine the effects of attention training on CBF and GM volume in healthy older adults. A comprehensive description of the interventions and the behavioral improvements resulting from this attention training program has been reported previously (Mozolic et al., 2009), and will not be the focus of this manuscript.

\section{ETHICS STATEMENT}

All participants gave written informed consent and were compensated approximately $\$ 20$ per hour for their participation in the study. All study procedures were approved by the Wake Forest University School of Medicine Institutional Review Board and conducted in accordance with the Declaration of Helsinki.

\section{PARTICIPANTS}

Seventy-five healthy older adults between the ages of 65 and 75 were recruited from the community and screened for eligibility in this study. Sixty-six participants were determined to be eligible for the study and were randomized to either the treatment or the control group. Due to scheduling constraints and MR incompatibility, only 58 subjects completed all scanning and training sessions and will be considered in the remainder of this report $\left(n_{\text {treatment }}=27, n_{\text {control }}=31\right.$, mean age $=69.5,31$ females). Technical limitations precluded the interpretation of perfusion data collected from 10 of the participants, so all CBF data reported are for 48 subjects $n_{\text {treatment }}=23$, $n_{\text {control }}=25$, mean age $=69.3,26$ females). Demographic data for participants is reported in Table 1.

Potential participants had to meet all of the following inclusion criteria for enrollment in the study: corrected visual acuity of $20 / 40$ or better in both eyes; normal color vision; hearing loss no greater than $50 \mathrm{~dB}$ at 1000 and $2000 \mathrm{~Hz}$; no evidence of dementia or cognitive impairment as indicated by a Mini-Mental Status Exam score within 2.5 standard deviations of the mean for their age and education level (Bravo and Hebert, 1997); no evidence of current substance abuse, indicated by a score below 10 on the Alcohol Use Disorders Identification Test (Bohn et al., 1995) and an evaluation of participant medical history; no evidence of depression, evaluated using the Medical Care Corporation survey ${ }^{1}$; no previous brain surgery, CNS trauma, neurological disorder, or use of antipsychotic or antiepileptic drugs, as determined by an evaluation of participant medical history.

Demographic data are presented for participants that completed all scanning visits of the study and for the subset of subjects included in the CBF analyses. Standard deviations are in parentheses, and $p$-values are from the results of two-tailed $t$-tests comparing the means of the treatment and control groups. There were no significant differences between the two groups in any of these demographic variables.

\section{DESIGN}

Once participants were enrolled and randomized into the study, they completed a pre-training MRI scan to evaluate resting CBF and anatomical structure. Participants then began 8 weeks of either

${ }^{1}$ www.mccare.com

Table 1 | Demographic data for study participants.

\begin{tabular}{llll}
\hline & \multicolumn{1}{c}{ Treatment } & Control & $\boldsymbol{p}$-Value \\
\hline IMAGING STUDY PARTICIPANTS & & \\
Age, years & $69.5(3.2)$ & $69.5(2.5)$ & 1.00 \\
Sex, \# females & 14 & 17 & \\
Education, years & $15.1(2.0)$ & $16.0(3.2)$ & 0.20 \\
MMSE, score & $28.3(1.6)$ & $28.5(1.9)$ & 0.80 \\
SUBSET INCLUDED IN CBF ANALYSES & & \\
Age, years & $69.3(3.2)$ & $69.4(2.4)$ & 0.95 \\
Sex, \# females & 12 & 14 & 0.11 \\
Education, years & $15.0(2.0)$ & $16.2(3.0)$ & 0.33 \\
MMSE, score & $28.3(1.6)$ & $28.7(1.6)$ & \\
\hline
\end{tabular}

The italicized values represents standard deviations. 
the treatment or control training program. Participants in both groups came to the laboratory for $1 \mathrm{~h}$ each week (total training time $=8 \mathrm{~h}$ ). After finishing their respective training programs, all participants completed a post-training MRI scan to re-evaluate CBF brain structure. All participants also completed pre- and posttraining cognitive evaluations.

\section{INTERVENTIONS}

An extensive description of the training interventions has been reported previously (Mozolic et al., 2009). Briefly, the treatment program was an individualized attention training program targeting auditory and visual attention that significantly decreased cross-modal distraction in this population. During training, participants practiced active suppression of irrelevant distractors while completing visual and auditory attention tasks. Visual and auditory tasks were paired with cross- and within-modality distractors. Tasks required detecting, classifying, and/or sequencing visual or auditory presentations of letters, words, and numbers while ignoring very salient auditory or visual distractors. Several of these task components were adapted from the commercially available APT-II program (Lash \& Associates Publishing/Training, Inc., Wake Forest, NC; Sohlberg and Mateer, 1987; Sohlberg et al., 2000; Lopez-Luengo and Vazquez, 2003). Distractors were series of short video clips (for visual distractors) or sound clips (for auditory distractors) of people, places, and events that provided distraction for the entire duration of each task (2-5 min). All task stimuli and distractors were presented to participants on a movie screen and overhead speakers via an LCD projector and participants made responses on each task by giving verbal or handwritten answers. Stimuli were sent to the LCD projector from a laptop computer with Presentation software ${ }^{2}$. A member of the study staff was always present in the training room to record responses, monitor performance, provide feedback, and control task presentation. Task difficulty for each participant increased adaptively, based on their performance on previous tasks. This design provided participants with repeated, continually challenging practice at suppressing distracting stimuli within and across modalities while completing demanding cognitive tasks.

The goal of the control program was to provide control participants with the same number and duration of laboratory visits, as well as similar interactions with study staff as participants in the treatment group, but without focused training on suppressing distractors. This was achieved using a small-group educational lecture program that required control participants to come into the laboratory $1 \mathrm{~h}$ each week for 8 weeks, just like the treatment group. Instead of completing the distractor tasks, however, the control group attended interactive lectures on various health topics (nutrition, heart disease, etc.). Administration of pre- and post-lecture quizzes, as well as weekly interactions with doctors, nurses, and therapists during these control sessions, helped maintain the single-blind design of the study. That is, participants were told that the purpose of the study was to evaluate the effects of two different training programs, and they were unaware that one training program had been designated the treatment group and the other was a control group.

${ }^{2}$ www.neurobs.com

\section{MRI EVALUATIONS}

During each MRI scan session, three different types of scans were performed: (1) a perfusion scan for evaluation of resting CBF; (2) a T-1 weighted, high-resolution structural scan; and (3) two BOLD fMRI scans of functional activity during cognitive tasks. The functional scans presented modified versions of a within-modality distraction task (Eriksen and Eriksen, 1974) and a cross-modal distraction task (Tellinghuisen and Nowak, 2003); however, due to design limitations and unanticipated effects attributed to the noise of the scanner environment, we were not able to detect behavioral changes on the within-modality distraction task, and the behavioral results from the cross-modal distraction task did not replicate previously published results from testing performed outside of the scanner (Tellinghuisen and Nowak, 2003; Mozolic et al., 2009), therefore only the perfusion and structural imaging results will be reported here.

The perfusion scan measured regional CBF using pulsed arterial spin labeling (PASL), a non-invasive MRI technique that uses magnetically labeled arterial blood water as an endogenous tracer for quantifying blood flow (Yang, 2002; Liu and Brown, 2007). By subtracting sequential images acquired with and without spin labeling, it is possible to obtain a measure of tissue perfusion (Wong et al., 1998). During the 8.5-min perfusion scan, participants rested quietly with their eyes open. Participants viewed a gray cross on a black background, presented through MR-compatible goggles with an integrated infrared eye tracker (Resonance Technology, Inc., Northridge, CA, USA) to ensure that subjects kept their eyes open throughout the experiment. A high-resolution anatomical image acquired for each participant was used to assess voxel-wise changes in GM volume with voxelbased morphometry (VBM).

\section{MRI ACQUISITION AND IMAGE PROCESSING}

All images were acquired with a 1.5-T GE scanner using a 4channel, quadrature phased array, neurovascular head coil (GE Medical Systems, Milwaukee, WI, USA). To assess CBF, the PASL scan employed Quantitative Imaging of Perfusion using a Single Subtraction with thin slice periodic saturation with a Flow-sensitive Alternating Inversion Recovery (Q2TIPS-FAIR) (Kim and Tsekos, 1997; Luh et al., 1999). Q2TIPS-FAIR acquires data in label/control pairs that are subtracted to generate a perfusion-weighted image. Imaging parameters are as follows: echo time $=28 \mathrm{~ms} ; \mathrm{TI}_{1}=800 \mathrm{~ms} ; \mathrm{TI}_{1 \mathrm{~s}}=1200 \mathrm{~ms} ; \mathrm{TI}=2000 \mathrm{~ms}$; $\mathrm{TR}=3000 \mathrm{~ms}$; receiver bandwidth $=62.5 \mathrm{kHz}$; flip angle $=90^{\circ}$; field of view $=24 \mathrm{~cm}$ (frequency) $\times 18 \mathrm{~cm}$ (phase); and acquisition matrix $=64 \times 48$ (11 slices, $8 \mathrm{~mm}$ thickness, $0 \mathrm{~mm}$ slice gap). A diffusion gradient with an equivalent $b$ value of $5.25 \mathrm{~mm}^{2} / \mathrm{s}$ is added to suppress intra-arterial spins (Yang et al., 1998). Prior to statistical analyses, motion correction was applied to the perfusion weighted volumes with a six-parameter rigid body transformation. After motion correction, the difference images were averaged together and quantitative perfusion maps were calculated from the equation:

$$
C B F=\frac{\Delta M\left(T I_{2}\right)}{2 M_{0, \text { blood }} \alpha T I_{1} q_{p}\left(T_{1, \text { tissue }}, T_{1, \text { blood }}, T I_{2}\right)} e^{\left(\frac{T I_{2}}{T_{1, \text { blood }}}\right)}
$$


where $\Delta \mathrm{M}\left(\mathrm{TI}_{2}\right)$ is the mean difference in signal intensity between label and control images, $M_{0, \mathrm{blood}}$ is the equilibrium magnetization of blood, $\alpha$ is the tagging efficiency, $\mathrm{TI}_{1}$ is the time duration of the tagging bolus, $\mathrm{TI}_{2}$ is the inversion time of each slice, $T_{1, \text { blood }}$ is the longitudinal relaxation time of blood, and $q_{\mathrm{p}}$ is a correction factor that accounts for the difference between T1 of blood and T1 of brain tissue (Wong et al., 1998). All other parameters are known or assumed to be constant $\left(\alpha=0.95, \mathrm{TI}_{1}=800 \mathrm{~ms}, T_{1, \text { blood }}=1200 \mathrm{~ms}\right)$. These quantitative CBF maps measure perfusion in each voxel in units of $\mathrm{ml}$ of blood/100 g of tissue/min. Quantitative CBF maps for each subject were normalized to the EPI template in SPM5 (Wellcome Department of Cognitive Neurology, London, www. fil.ion.ucl.ac.uk/spm/software/spm5/).

High-resolution, T1-weighted structural scans were obtained using an inversion recovery $3 \mathrm{D}$ spoiled gradient echo sequence (matrix size $=256 \times 256$; field of view $=24 \mathrm{~cm} ; 1.5 \mathrm{~mm}$ sections, no gap; 128 slices; in-plane resolution $=0.94 \mathrm{~mm}$ ). To assess local changes in tissue volume, we used VBM, implemented in SPM5. This method segments each participant's structural image into tissue types (GM, WM, and CSF), performs bias correction (for image intensity inhomogeneities), and spatially normalizes the image to the standard MNI template in a unified, iterative process (Ashburner and Friston, 2005). To investigate the absolute volume of GM rather than relative concentrations, voxel values were modulated by the Jacobian determinants derived during the spatial normalization (Good et al., 2001).

\section{STATISTICAL ANALYSIS}

All statistical analyses were performed in SPM5, implemented in Matlab (Mathworks, Inc., Sherborn, MA, USA). To compare training-induced changes in regional CBF between the groups, we first created a perfusion difference map for each subject by subtracting their pre-training image from their post-training image. These difference images were smoothed with an $8 \times 8 \times 10 \mathrm{~mm}$ Gaussian kernel. A two-sample $t$-test was then used to compare voxel-wise differences in CBF changes between the treatment and control groups. Results were thresholded at $p<0.001$ with an extent correction of $p<0.05$. To confirm that any changes in CBF were local, rather than global, we also performed a $2_{\text {group }} \times 2_{\text {session }}$ mixed ANOVA on whole-brain average GM perfusion values. $A$-value less than 0.05 was considered statistically significant for this analysis.

We followed a similar procedure to evaluate group differences in GM volume changes. Difference maps were created by subtracting each participant's modulated, normalized, segmented GM image pre-training image from the post-training one. These images were then smoothed with an 8-mm isotropic Gaussian kernel. A two-sample $t$-test compared differences in training-induced GM changes between the two groups. In addition to the whole-brain VBM analyses, we also evaluated GM changes in a region of interest (ROI) created from the group CBF analysis. This ROI was defined by a binary mask of all significant voxels found in the whole-brain group comparison of CBF changes. A $2_{\text {group }} \times 2_{\text {session }}$ ANOVA was used to assess changes in GM volume in the ROI.

To determine whether there was a significant relationship between regional CBF change and alterations in GM structure with training, we performed a voxel-wise correlation analysis between CBF difference images and GM difference images for each participant using the Biological Parametric Mapping (BPM) toolbox (Casanova et al., 2007). BPM utilizes the general linear model to perform multimodal image analyses on a voxel-by-voxel basis, so this analysis completed a unique correlation between the perfusion image and the anatomical GM image at each voxel.

To assess the relationship between the imaging results and behavioral improvements following attention training, we also performed a correlation between perfusion change values extracted from the CBF ROI (defined above) and response time interference on a crossmodal distraction task performed by participants during pre- and post-training cognitive testing. Performance on this task was the primary outcome measure used in the behavioral evaluation of the training program and shows significant improvement with training (Mozolic et al., 2009). In this task, auditory distractors can interfere with responses to visual targets. After completion of the training program, however, the treatment group demonstrated significant reductions in interference, relative to controls [for further details on behavioral task design and results, please see: (Mozolic et al., 2009)]. Therefore, we expected larger training-induced increases in regional CBF among participants who showed greater reductions cross-modal distraction.

Finally, to explore the power of our findings and plan for future studies, we calculated voxel-wise power maps to depict the statistical power and number of subjects needed to detect training-induced changes in CBF in regions that did not achieve significance in the current study. Power maps were based on the effect size produced by the following group contrast: $\Delta \mathrm{CBF}_{\text {treatment }}>\Delta \mathrm{CBF}_{\text {control }}$. In each voxel, we performed two calculations: (1) the number of subjects required in each group to detect a significant finding with $80 \%$ power and $p<0.05$ with a family-wise error rate (FWE) correction for multiple comparisons; and (2) the power $(1-\beta)$ to detect significant changes, given $n=50$ subjects in each group. These analyses generated one map where the intensity in each voxel represents the number of subjects that would be required to detect a significant finding in that voxel, and a second map where the intensity represents the power that would be achieved in that voxel if the study included 50 subjects per group.

\section{RESULTS}

As stated above, a complete report on the behavioral improvements resulting from the attention training program has been previously published (Mozolic et al., 2009); however, to ensure that behavioral results from the subset of participants included in this imaging study are reflective of those previously reported for the entire group, we confirmed the main behavioral findings in this group of 23 treatment and 25 control participants. Analyses indicated that significant training-induced reductions in cross-modal interference remained present in this subset of participants, despite the reduced sample size: significant Group ${ }_{\text {(treatment, control) }} \times \operatorname{Session}_{\text {(pre- }}$ training, post-training $)$ interaction $\left(F_{1,46}=4.24, p<0.05\right)$. Additionally, after training, this subset of participants demonstrated a significant improvement in their ability to suppress multisensory distraction during selective visual attention $\left(t_{22}=3.31, p<0.003\right)$ and during selective auditory attention $\left(t_{22}=2.94, p<0.007\right)$. This reanalysis of the data demonstrates that the subset of participants on whom we collected CBF data was not qualitatively different from the larger group analyzed extensively in the previously published 
report. These participants responded to the attention training program in the same way as the larger group, with decreases in distractibility and an improved ability to ignore cross-modal sensory noise.

In a voxel-wise analysis of CBF, the treatment group had significantly larger training-induced increases in perfusion than controls in right inferior frontal cortex (IFC; peak voxel MNI coordinates: 40, 48, -15; Figure 1A). Mean perfusion values extracted from this cluster increased from 42.6 to $59.2 \mathrm{ml} / 100 \mathrm{~g}$ tissue/min for the treatment group, and decreased slightly for the control group, from 58.1 to $54.2 \mathrm{ml} / 100 \mathrm{~g}$ tissue/min (Figure 1B).
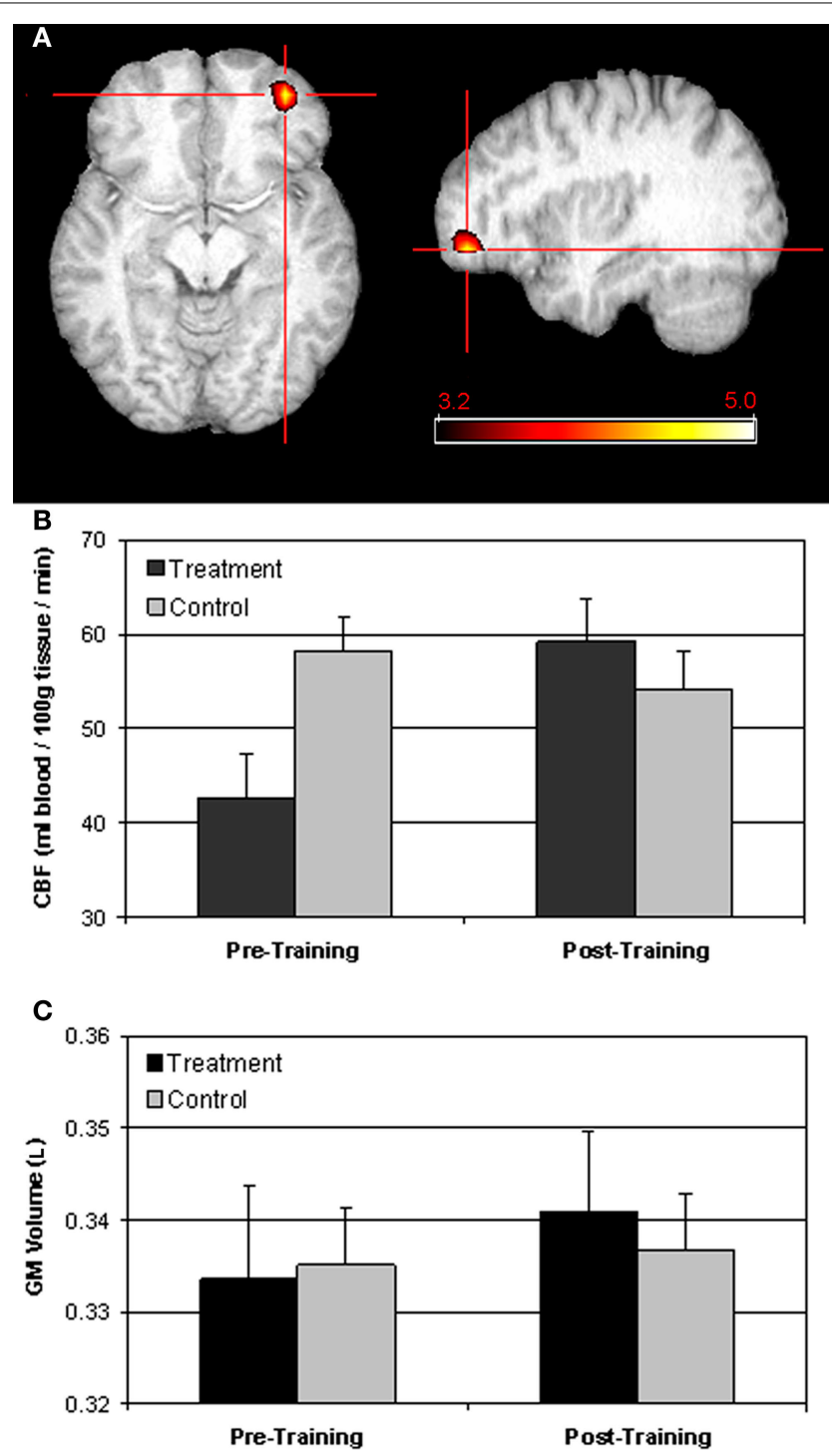

FIGURE 1 | Training-related changes in resting CBF. Whole-brain voxel-wise analysis of training-induced changes in CBF indicated that the treatment program produced larger increases in CBF to the right inferior prefrontal cortex than the control program (A). Quantitative perfusion values extracted from the region shown in $(\mathbf{A})$ increased from 43.6 to $59.2 \mathrm{ml} / 100 \mathrm{~g} / \mathrm{min}$ for the treatment group and decreased slightly, from 58.1 to $54.2 \mathrm{ml} / 100 \mathrm{~g} / \mathrm{min}$ for the control group (B). There were no significant changes in GM volume in this region for either the treatment group or controls (C).
As illustrated in Figure 1, between-group differences in $\mathrm{CBF}$ change were driven by the treatment group, where mean perfusion values increased after training. Although this training-induced increase served to bring CBF in the treatment group up to the level of the control group, rather than to exceed CBF in the control group, these increases in blood flow are still an important result of the training procedure. To further explore the impact of the treatment and control programs we also compared whole-brain $\mathrm{CBF}$ in the two groups at the pre-training and post-training time points. Importantly, there were no voxels that were significantly different between the two groups at either time point. So although a post-hoc analysis of CBF values in the right IFC indicated a slight baseline difference in blood flow between the treatment and control groups $\left(t_{46}=2.62, p<0.01\right)$, the a priori, whole-brain, voxel-wise analysis indicated that there were no significant baseline differences in CBF between the two groups. When the threshold was lowered to explore trends in the data $(p<0.001$, uncorrected), there was a region of the left IFC (peak voxel: $-12,40,-20$ ) that showed lower $\mathrm{CBF}$ for the treatment group relative controls prior to training, however this region did not overlap with voxels that showed significantly larger training-induced increases in CBF for treatment group. Post-training, there were no differences between the two groups at this lowered threshold. When the treatment group and control group were each analyzed separately, there were no brain regions that showed significant pre- to post-training increases for either group; however, there were sub-threshold ( $p<0.001$, uncorrected) increases in CBF to the left ACC (peak voxel: $-8,36,-10$ ) and the right IFC (peak voxel: $24,48,-10$ ) after the treatment intervention. No sub-threshold changes were observed for the control group. These sub-threshold results are reported to aid in the overall interpretation of the findings; however, only the between-group comparison of training-induced changes in regional CBF, reported at the beginning of this section, yielded significant findings.

The analysis of whole-brain average GM perfusion yielded no significant effects of group or session and no significant group $\mathrm{x}$ session interaction (all $F_{1,46}<2.0$, all $p>0.05$ ). These results suggest that larger increases in regional CBF for the treatment group, relative to controls, reflect local changes in perfusion and are not an artifact of global differences in blood flow.

In a whole-brain evaluation of GM volume, there were no areas of significant difference between the treatment and control groups. We also restricted our analysis of GM volume changes to the brain areas that showed training-induced increases in CBF. However, this ROI analysis yielded no significant effects of group or session, and no significant group $\times$ session interaction $\left(\right.$ all $F_{1,55}<2.7$, all $p>0.10$; Figure 1C). To further investigate any relationship between changes in $\mathrm{CBF}$ and $\mathrm{GM}$ volume, we performed a voxel-wise correlation analysis for each between pre-to-post CBF values and pre-to-post GM volumes for each participant. We found no significant correlation in any brain region between training-induced changes in perfusion and GM volume. These results indicate that increased $\mathrm{CBF}$ to the right IFC following cognitive training was not accompanied by measureable alterations in GM volume.

A correlation analysis of CBF changes and behavioral outcomes following training indicated that there was a modest relationship between increased perfusion in the right IFC ROI and reduced cross-modal distraction (Pearson's $r_{21}=-0.32, p<0.07$; 
Figure 2). Although all outliers are removed from the behavioral data during analysis and there were no outlying points in the $\mathrm{CBF}$ data ( \pm 3 standard deviations), to insure that this correlation was not driven solely by the participants with the largest increases in blood flow, we removed the three highest CBF values. This analysis still yielded a modest correlation between increased CBF and reduced cross-modal distraction (Pearson's $r_{18}=-0.30$, $p<0.10)$. There was no significant correlation between $\mathrm{CBF}$ and distractibility for the control group (Pearson's $r_{23}=0.11$, $p=\mathrm{n} . \mathrm{s})$. Importantly, these data suggest that participants who demonstrated larger increases in perfusion following attention training were also likely to be better at suppressing distracting cross-modal stimuli.

Voxel-wise power calculations based on the effect size observed in this study were used to generate a map of the number of subjects per group that would be required to detect significant traininginduced increased in regional $\mathrm{CBF}$ (at $80 \%$ power, $p<0.05 \mathrm{FWE}$ correction; Figure 3A). This map demonstrates that the addition of several subjects in each group could reveal further areas of $\mathrm{CBF}$ increase, including bilateral IFC, rostral ACC, and medial frontal cortex (MFC). A second map generated using the voxelwise power calculations indicated that the statistical power to detect training-induced effects, given $n=50$ subjects per group, would be near $70 \%$ for regions in right IFC, rostral ACC, and MFC (Figure 3B).

\section{DISCUSSION}

The data presented here demonstrate that a cognitive training program aimed at reducing distractibility in older adults produces larger increases in resting $\mathrm{CBF}$ than a control program. These are, to our knowledge, the first data demonstrating that a cognitive training intervention can improve resting perfusion

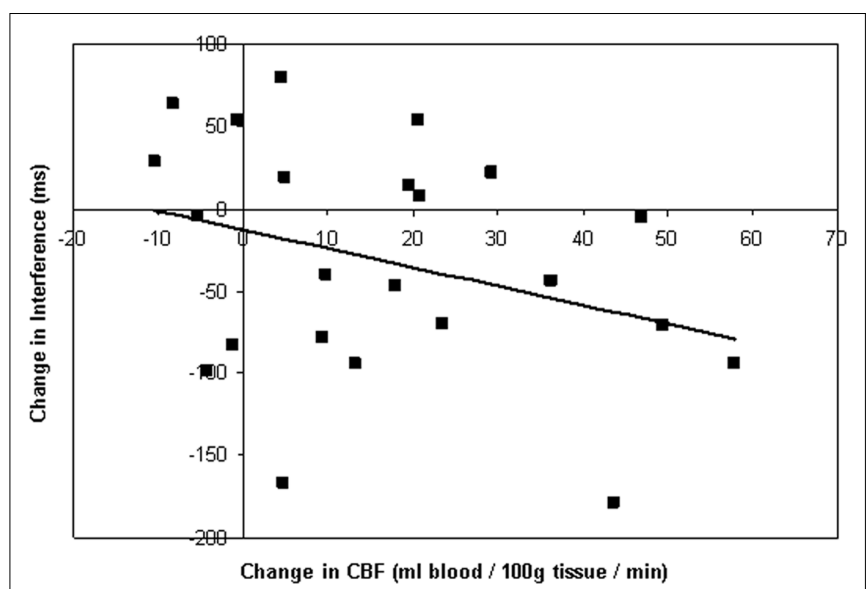

FIGURE 2 | Relationship between changes in CBF and behavioral performance. Training-induce increases in resting CBF in the right inferior PFC were modestly correlated with behavioral reductions in cross-modal interference after training. Analysis of individual perfusion change values (extracted from the region depicted in Figure 1A) and changes in the response time to visual targets during auditory distraction yielded a marginally significant negative correlation (Pearson's $r=-0.32, p<0.07$ ). Decreases in response time to visual targets during auditory distraction were indicative of reductions in behavioral interference.

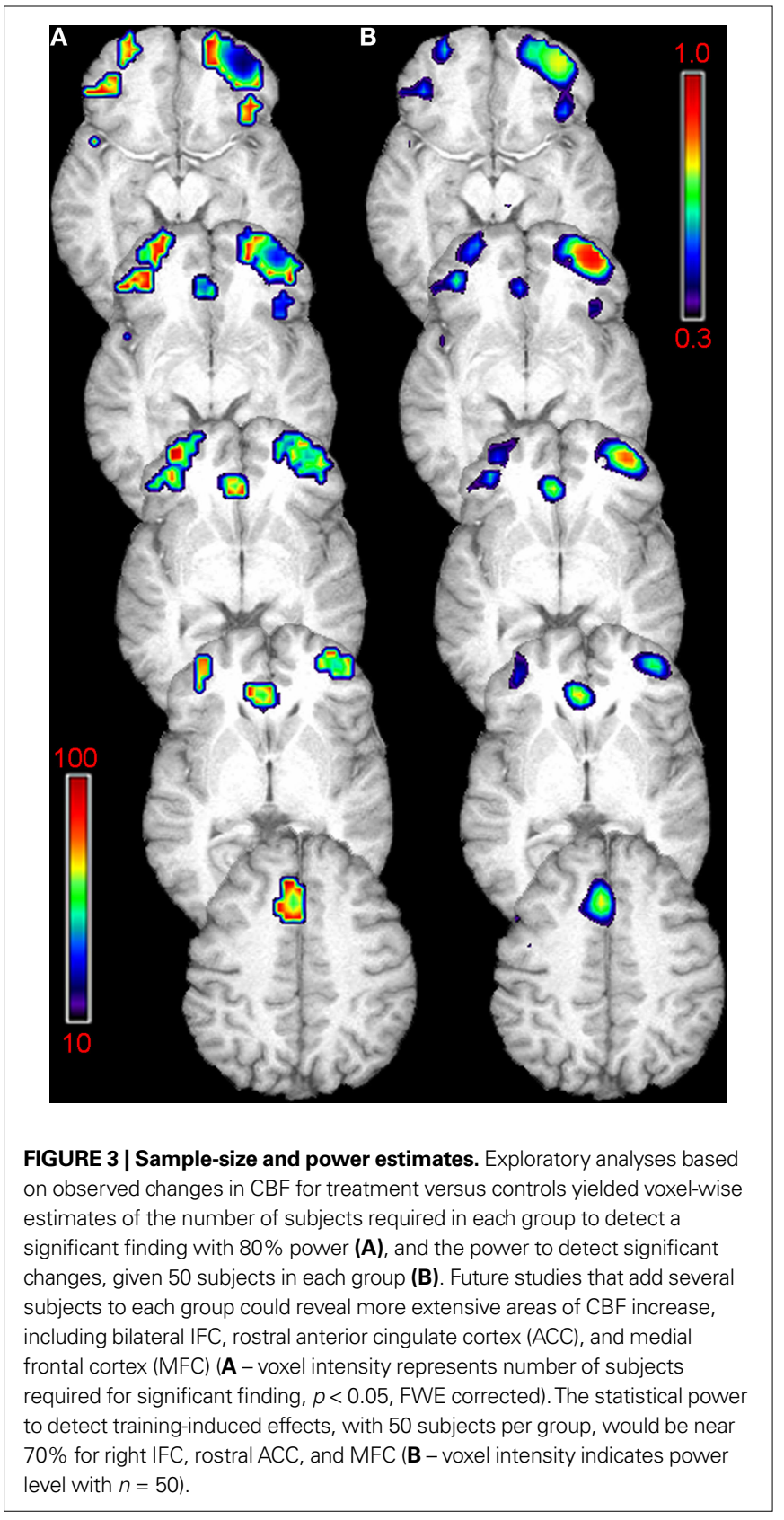

rates in healthy older adults. In a whole-brain analysis of traininginduced changes, the right IFC exhibited larger CBF increases in the treatment group than in the control group. Further, there was a modest correlation between enhanced $\mathrm{CBF}$ in this region and reduced cross-modal distraction following training, suggesting an important link between perfusion increases and behavioral improvements.

One potential concern with these results is that, due to slightly lower baseline CBF levels in the right IFC region for the treatment group than for controls, increases in $\mathrm{CBF}$ for the treatment group raised CBF levels to those of the control group, rather than to levels that exceeded the control group. Although regression to the mean is one possible explanation for these results, several factors indicate that increased resting $\mathrm{CBF}$ for the treatment group is not 
simply an artifact of lower baseline CBF. First, in a whole-brain voxel-wise analysis of pre-training perfusion levels, there were no brain regions that showed significant between-group differences. This finding indicates that there were no detectable baseline differences between the two groups when looking across the entire brain. Additionally, there were no pre-training differences between the two groups in the behavioral cross-modal distraction task, yet the treatment group showed larger reductions in cross-modal distraction than controls and reductions in distraction were modestly correlated with increased resting CBF in the right IFC. Nevertheless, a larger sample size would likely improve the randomization procedure and reduce the likelihood of baseline differences between the two groups.

The rostral inferior region of the PFC (corresponding with Brodmann's Area 10) that exhibited training-induced CBF increases is highly interconnected with other areas of the PFC, as well as the cingulate cortex (Barbas and Pandya, 1989; Arikuni et al., 1994; Ramnani and Owen, 2004). Cells of the rostral PFC also have a very high density of dendritic spines relative to other areas of the PFC, suggesting that this area plays an important role in integrating incoming information (Jacobs et al., 2001; Ramnani and Owen, 2004). Additionally, this region has been implicated in a number of the functions required for successful completion of the training tasks used in this program, including maintenance of attentional set, prospective memory, and multi-tasking (Koechlin et al., 1999; Burgess et al., 2001,2007; Velanova et al., 2003; Dreher et al., 2008). For example, a representative training task visually presented a series of words during ongoing auditory noise. Participants were required to remember to press a buzzer only on target words (i.e. words that contained one more letter than the previous words) and to ignore salient auditory distractors. Nearly all tasks required participants to remember to perform one or a series of actions when the appropriate cue was detected and to maintain focus on several different complex tasks in the face of distracting information. Repeated engagement of rostral frontal cortex networks during such training tasks that exercised prospective memory, attentional control, and multi-tasking may have altered synaptic connectivity or metabolic demand and resulted in the observed increase in resting perfusion rates. Additionally, BA10 has also been implicated, along with the medial temporal and visual cortices, in the generation of spontaneous thought (Christoff et al., 2004). Interestingly, our results do not show any training-induced differences in temporal or visual CBF; however, analysis of subthreshold results (Figure 3), shows group differences in medial frontal and anterior cingulate cortices, brain regions typically associated with limiting distraction and cognitive control (Weissman et al., 2004; Botvinick, 2007). These results, along with behavioral improvements in task performance, indicate that increases in IFC activity after training are related to changes in attentional control mechanisms, rather than differences in spontaneous thought processes. Incorporating verbal reports or other measures of behavior during resting scans could help to clarify this issue in future studies.

A recent study by Mazoyer et al. (2009) demonstrated that in young adults, cognitive training on reasoning bias improved performance on logical reasoning problems and increased CBF at rest. Importantly, these changes were noted on the same day, after a single 30- minute training session and included increased CBF during rest in the IFC and anterior cingulate, as well as other areas of the PFC and the thalamus (Mazoyer et al., 2009). These findings in young adults parallel the results presented here in older adults and indicate that changes in resting perfusion rates can occur almost immediately following the acquisition of new skills or knowledge.

The results of the present study are also in accord with data from a number of experiments demonstrating that increased frontal activity corresponds with improved outcomes for older adults on a wide range of cognitive tasks (Grady, 1996; Reuter-Lorenz et al., 2000; Cabeza et al., 2002, 2004; Langenecker and Nielson, 2003; Grady et al., 2005); although see also (Colcombe et al., 2005; Rypma et al., 2005). For example, Cabeza et al., (2002) have shown that older adults who exhibited increased bilateral PFC activity compared to young adults during source memory retrieval also displayed superior performance on this task relative to older adults who did not increase bilateral frontal activity. Increased regional CBF observed in our study may be a compensatory mechanism activated by training to overcome neural or metabolic declines in the PFC, although evidence for this hypothesis is limited by the lack of a young control group in this study.

Another point to consider is that the standard resting condition used in this study and in many others (e.g., Raichle et al., 2001; Uludag et al., 2004) could have been approached differently by the treatment and control participants. The resting condition required subjects to maintain fixation on a cross during the presence of scanner noise, and thus the treatment group could have been employing practiced skills in attention and suppression of distracting stimuli. However, we observed no differences between the two groups in $\mathrm{CBF}$ to the visual or auditory cortices, areas that would be expected to show increases and decreases in activity, respectively, if the treatment group was actively focusing visual attention and suppressing auditory activity. Additionally, due to the fact that increases in $\mathrm{CBF}$ were correlated with improvements in behavioral performance, our results suggest that training influenced not only resting activity, but also task-related activity. Additional studies incorporating fMRI during attentionally-demanding tasks will be important for determining the relationship between training-induced changes in rest- and task-related blood flow and brain activity.

Because this was a relatively small pilot study powered to detect behavioral changes associated with training, we also examined the power of the perfusion finding and the sample size required to detect changes in regions that did not achieve significance in this study. These exploratory analyses indicated that sub-threshold increases in CBF extended bilaterally in the rostral IFC and also included regions of the ACC. Thus, future studies with larger sample sizes may be able to detect more widespread increases in perfusion after cognitive training.

In contrast to the perfusion results and our initial hypothesis, we observed no significant change in GM volume following training. Voxel-wise correlations between $\Delta \mathrm{CBF}$ and $\Delta \mathrm{GM}$ volume also indicated that there was no significant relationship between perfusion changes and alterations in brain structure. These results suggest that training-induced increases in resting CBF were not accompanied by any detectable growth of cortical or subcortical tissue. It is possible that our study did not continue long enough or was underpowered to detect changes in GM volume. Based on our findings, however, it is possible that the cerebral vasculature 
response to cognitive training may be a more sensitive marker for evaluating short-term interventional outcomes than VBM. Although previous studies have demonstrated increases in tissue volume following training in older adults, these interventions involved aerobic exercise (Colcombe et al., 2006) or skill learning (Boyke et al., 2008), rather than cognitive training. Animal models indicate that environmental enrichment, which typically encourages both motor activity and spatial learning, can promote neuronal proliferation and survival in the hippocampus as well as dendritic branching in the cerebellum of aging rodents (Greenough et al., 1986; Churchill et al., 2002; Kempermann et al., 2002). It may be possible that cognitive training alone is not sufficient to produce noticeable changes in GM volume; however, combining practice on cognitive and motor tasks with cardiovascular fitness training regimens could yield larger results than any individual component.

In conclusion, the results of this study demonstrate that a cognitive training program that reduces cross-modal distraction in older adults also produces larger increases in CBF to the PFC than a control program. These changes were not accompanied by alterations in tissue volume, but were modestly correlated with behavioral improvements, suggesting that CBF may be a more sensitive marker of training-induced improvements than GM volume. Future trials that integrate various training modalities, valid measures of task-

\section{REFERENCES}

Alain, C., and Woods, D. L. (1999). Agerelated changes in processing auditory stimuli during visual attention: evidence for deficits in inhibitory control and sensory memory. Psychol. Aging 14, 507-519.

Arikuni, T., Sako, H., and Murata, A. (1994). Ipsilateral connections of the anterior cingulate cortex with the frontal and medial temporal cortices in the macaque monkey. Neurosci. Res. 21, 19-39.

Ashburner, J., and Friston, K. J. (2005). Unified segmentation. Neuroimage 26, 839-851.

Asllani, I., Habeck, C., Borogovac, A., Brown, T. R., Brickman, A. M., and Stern, Y. (2009). Separating function from structure in perfusion imaging of the aging brain. Hum. Brain Mapp. 30, 2927-2935.

Ball, K., Berch, D. B., Helmers, K. F., Jobe, J. B., Leveck, M. D., Marsiske, M., Morris, J. N., Rebok, G. W., Smith, D. M., Tennstedt, S. L., Unverzagt, F. W., and Willis, S. L. (2002). Effects of cognitive training interventions with older adults: a randomized controlled trial. JAMA 288, 2271-2281.

Bangen, K. J., Restom, K., Liu, T. T., Jak, A. J., Wierenga, C. E., Salmon, D. P., and Bondi, M. W. (2007). Differential age effects on cerebral blood flow and BOLD response to encoding: associations with cognition and stroke risk. Neurobiol. Aging 30, 1276-1287.
Barbas, H., and Pandya, D. N. (1989). Architecture and intrinsic connections of the prefrontal cortex in the rhesus monkey. J. Comp. Neurol. 286, 353-375.

Beason-Held, L. L, Kraut, M. A., and Resnick, S. M. (2009). Stability of default-mode network activity in the aging brain. Brain Imaging Behav. 3, 123-131.

Bentourkia, M., Bol, A., Ivanoiu, A., Labar, D., Sibomana, M., Coppens, A., Michel, C., Cosnard, G., and De Volder, A. G. (2000). Comparison of regional cerebral blood flow and glucose metabolism in the normal brain: effect of aging. J. Neurol. Sci. 181, 19-28. Walter, C., Khan, R., and Naumann, E. (2009). Resting cerebral blood flow, attention, and aging. Brain Res. 1267, 77-88.

Bherer, L., Kramer, A. F., Peterson, M. S., Colcombe, S., Erickson, K., and Becic, E. (2008). Transfer effects in task-set cost and dual-task cost after dual-task training in older and younger adults: further evidence for cognitive plasticity in attentional control in late adulthood. Exp. Aging Res. 34, 188-219.

Bohn, M. J., Babor, T. F., and Kranzler, H. R. (1995). The Alcohol Use Disorders Identification Test (AUDIT): validation of a screening instrument for use in medical settings. J. Stud. Alcohol $56,423$.
Bertsch, K., Hagemann, D., Hermes, M.,

related activity (fMRI), and/or larger study populations may generate more widespread functional and structural brain changes and additional functional gains for older adults. Finally, the addition of a control group of young subjects could determine whether training-induced changes are specific to older participants or could benefit younger adults as well.

\section{ACKNOWLEDGMENTS AND DISCLOSURE}

The authors would like to thank Ashley Morgan, Melissa RawleyPayne, Ashley Long, the Center for Biomolecular Imaging, and Drs. Robert Kraft and Jonathan Burdette for their invaluable assistance with study design and data collection. We would also like to thank the two reviewers for their helpful suggestions. This work was funded by the National Institutes of Health (grant numbers: AG030838 to Jennifer L. Mozolic, NS059793 to Satoru Hayasaka, AG026353 and NS42568 to Paul J. Laurienti, and RR07122 to the Wake Forest University General Clinical Research Center) and the Roena Kulynych Memory and Cognitive Research Center. The funders had no role in study design, data collection and analysis, decision to publish, or preparation of the manuscript. Jennifer L. Mozolic designed the study, collected and analyzed the data, and wrote the manuscript. Satoru Hayasaka performed the power analyses and created the power maps. Paul J. Laurienti designed the study, acted as an advisor on the project, and edited the manuscript.

Botvinick, M. M. (2007). Conflict monitoring and decision making: reconciling two perspectives on anterior cingulate function. Cogn. Affect. Behav. Neurosci. 7, 356-366.

Boyke, J., Driemeyer, J., Gaser, C., Buchel C., and May, A. (2008). Traininginduced brain structure changes in the elderly. J. Neurosci. 28, 7031-7035.

Bravo, G., and Hebert, R. (1997). Age- and education-specific reference values for the Mini-Mental and modified MiniMental State Examinations derived from a non-demented elderly population. Int. J. Geriatr. Psychiatry 12, 1008-1018.

Buckner, R. L. (2004). Memory and executive function in aging and $\mathrm{AD}$ : multiple factors that cause decline and reserve factors that compensate. Neuron 44, 195-208.

Burgess, P. W., Gilbert, S. J., and Dumontheil, I. (2007). Function and localization within rostral prefrontal cortex (area 10). Philos. Trans. R. Soc. Lond., B, Biol. Sci. 362, 887-899.

Burgess, P. W., Quayle, A., and Frith, C. D. (2001). Brain regions involved in prospective memory as determined by positron emission tomography. Neuropsychologia 39, 545-555.

Burke, S. N., and Barnes, C. A. (2006). Neural plasticity in the ageing brain. Nat. Rev. Neurosci. 7, 30-40.

Buschkuehl, M., Jaeggi, S. M., Hutchison, S., Perrig-Chiello, P., Däpp, C., Müller, M., Breil, F., Hoppeler, H., and Perrig, W. J. (2008). Impact of working memory training on memory performance in old-old adults. Psychol. Aging 23, 743.

Cabeza, R., Anderson, N. D., Locantore, J. K., and McIntosh, A. R. (2002). Aging gracefully: compensatory brain activity in high-performing older adults. Neuroimage 17, 1394.

Cabeza, R., Daselaar, S. M., Dolcos, F., Prince, S. E., Budde, M., and Nyberg, L. (2004). Task-independent and taskspecific age effects on brain activity during working memory, visual attention and episodic retrieval. Cereb. Cortex 14, 364-375.

Casanova, R., Srikanth, R., Baer, A., Laurienti, P. J., Burdette, J. H., Hayasaka, S., Flowers, L., Wood, F., and Maldjian, J.A. (2007). Biological parametric mapping: a statistical toolbox for multimodality brain image analysis. Neuroimage 34, 137-143.

Christoff, K., Ream, J. M., and Gabrieli, J. D. (2004). Neural basis of spontaneous thought processes. Cortex 40 , 623-630.

Churchill, J. D., Galvez, R., Colcombe, S., Swain, R. A., Kramer, A. F., and Greenough, W. T. (2002). Exercise, experience and the aging brain. Neurobiol. Aging 23, 941-955.

Colcombe, S. J., Erickson, K. I., Scalf, P. E., Kim, J. S., Prakash, R., McAuley, E., Elavsky, S., Marquez, D. X., Hu, L., and Kramer, A. F. (2006). Aerobic exercise training increases brain volume in aging humans. J. Gerontol. A Biol. Sci. Med. Sci. 61, 1166-1170. 
Colcombe, S. J., Kramer, A. F., Erickson, K. I., and Scalf, P. (2005). The implications of cortical recruitment and brain morphology for individual differences in inhibitory function in aging humans. Psychol. Aging 20, 363-375.

Colcombe, S. J., Kramer, A. F., Erickson, K. I., Scalf, P., McAuley, E., Cohen, N. J., Webb, A., Jerome, G. J., Marquez, D. X., and Elavsky, S. (2004). Cardiovascular fitness, cortical plasticity, and aging. Proc. Natl. Acad. Sci. U.S.A. 101, 3316-3321.

Corbetta, M., and Shulman, G. L. (2002). Control of goal-directed and stimulusdriven attention in the brain. Nat. Rev . Neurosci. 3, 201-215.

Dahlin, E., Nyberg, L., Backman, L., and Neely, A. S. (2008a). Plasticity of executive functioning in young and older adults: immediate training gains, transfer, and long-term maintenance. Psychol. Aging 23, 720.

Dahlin, E., Neely, A. S., Larsson, A., Backman, L., and Nyberg, L. (2008b). Transfer of learning after updating training mediated by the striatum. Science 320, 1510-1512.

Draganski, B., Gaser, C., Busch, V., Schuierer, G., Bogdahn, U., and May, A. (2004). Neuroplasticity: changes in grey matter induced by training. Nature 427, 311-312.

Draganski, B., Gaser, C., Kempermann, G., Kuhn, H. G., Winkler, J., Buchel, C., and May, A. (2006). Temporal and spatial dynamics of brain structure changes during extensive learning. J. Neurosci. 26, 6314-6317.

Dreher, J. C., Koechlin, E., Tierney, M., and Grafman, J. (2008). Damage to the fronto-polar cortex is associated with impaired multitasking. PLoS ONE 3:e3227. doi: 10.1371/journal. pone. 0003227.

Erickson, K. I., Colcombe, S. J., Wadhwa, R., Bherer, L., Peterson, M. S., Scalf, P. E., Kim, J. S., Alvarado, M., and Kramer,A. F. (2007). Training-induced plasticity in older adults: effects of training on hemispheric asymmetry. Neurobiol. Aging 28, 272-283.

Eriksen, B. A., and Eriksen, C. W. (1974). Effects of noise letters upon the identification of a target letter in a nonsearch task. Percept. Psychophys. 16, 143-149.

Fassbender, C., Murphy, K., Foxe, J. J., Wylie, G. R., Javitt, D. C., Robertson, I. H., and Garavan, H. (2004). A topography of executive functions and their interactions revealed by functional magnetic resonance imaging. Brain Res. Cogn. Brain Res. 20, 132.

Fjell, A. M., Westlye, L. T., Amlien, I., Espeseth, T., Reinvang, I., Raz, N., Agartz, I., Salat, D. H., Greve, D. N., Fischl, B., Dale, A. M., and Walhovd, K. B. (2009). High consistency of regional cortical thinning in aging across multiple samples. Cereb. Cortex 19, 2001-2012.

Golestani, N., and Zatorre, R. J. (2004). Learning new sounds of speech: reallocation of neural substrates. Neuroimage 21, 494.

Good, C. D., Johnsrude, I. S., Ashburner, J., Henson, R. N., Friston, K. J., and Frackowiak, R. S. (2001). A voxelbased morphometric study of ageing in 465 normal adult human brains. Neuroimage 14, 21-36.

Grady, C. L. (1996). Age-related changes in cortical blood flow activation during perception and memory. Ann. N. Y. Acad. Sci. 777, 14-21.

Grady, C. L., McIntosh, A. R., and Craik, F. I. (2005). Task-related activity in prefrontal cortex and its relation to recognition memory performance in young and old adults. Neuropsychologia 43 , 1466-1481.

Greenough, W. T., McDonald, J. W., Parnisari, R. M., and Camel, J. E. (1986). Environmental conditions modulate degeneration and new dendrite growth in cerebellum of senescent rats. Brain Res. 380, 136-143.

Hugenschmidt, C. E., Mozolic, J. L., and Laurienti, P. J. (2009). Suppression of multisensory integration by modalityspecific attention in aging. Neuroreport 20, 349-353.

Hugenschmidt, C. E., Peiffer, A. M., Kraft, R. A., Casanova, R., Deibler, A. R., Burdette, J. H., Maldjian, J. A., and Laurienti, P. J. (2008). Relating imaging indices of white matter integrity and volume in healthy older adults. Cereb. Cortex 18, 433-442.

Hyde, K. L., Lerch, J., Norton, A., Forgeard, M., Winner, E., Evans, A. C., and Schlaug, G. (2009). Musical training shapes structural brain development. J. Neurosci. 29, 3019-3025.

Ilg, R., Wohlschlager, A. M., Gaser, C., Liebau, Y., Dauner, R., Woller, A., Zimmer, C., Zihl, J., and Muhlau, M. (2008). Gray matter increase induced by practice correlates with taskspecific activation: a combined functional and morphometric magnetic resonance imaging study. J. Neurosci. 28, 4210-4215.

Isingrini, M., Perrotin, A., and Souchay, C. (2008). Aging, metamemory regulation and executive functioning. Prog. Brain Res. 169, 377-392.

Jacobs, B., Schall, M., Prather, M., Kapler, E., Driscoll, L., Baca, S., Jacobs, J., Ford, K., Wainwright, M., and Treml, M. (2001). Regional dendritic and spine variation in human cerebral cortex: a quantitative golgi study. Cereb. Cortex $11,558-571$.

Jennings, J., Webster, L., Kleykamp, B., and Dagenbach, D. (2005). Recollection training and transfer effects in older adults: successful use of a repetitionlag procedure. Aging Neuropsychol. Cogn. Neuropsychol. Dev. Cogn. 12, 278-298.

Jessberger, S., and Gage, F. H. (2008). Stem-cell-associated structural and functional plasticity in the aging hippocampus. Psychol. Aging 23 684-691.

Kempermann, G., Gast, D., and Gage, F. H. (2002). Neuroplasticity in old age: sustained fivefold induction of hippocampal neurogenesis by longterm environmental enrichment. Ann. Neurol. 52, 135-143.

Kim, S. G., and Tsekos, N. V. (1997) Perfusion imaging by a flow-sensitive alternating inversion recovery (FAIR) technique: application to functional brain imaging. Magn. Reson. Med. 37, 425-435.

Koechlin, E., Basso, G., Pietrini, P., Panzer, S., and Grafman, J. (1999). The role of the anterior prefrontal cortex in human cognition. Nature 399 148-151.

Kramer, A. F., Bherer, L., Colcombe, S. J., Dong, W., and Greenough, W. T. (2004). Environmental influences on cognitive and brain plasticity during aging. J. Gerontol. A Biol. Sci. Med. Sci. 59, M940.

Kramer, A. F., Larish, J. F., and Strayer, D. L. (1995). Training for attentional control in dual task settings: a comparison of young and old adults. J. Exp. Psychol. Appl. 1, 50-76.

Langenecker, S. A., and Nielson, K. A. (2003). Frontal recruitment during response inhibition in older adults replicated with fMRI. Neuroimage 20, 1384 .

Liu, T. T., and Brown, G. G. (2007). Measurement of cerebral perfusion with arterial spin labeling: part 1 . Methods. J. Int. Neuropsychol. Soc. 13 , 517-525.

Lopez-Luengo, B., and Vazquez, C. (2003). Effects of attention process training on cognitive functioning of schizophrenic patients. Psychiatry Res, 119, 41-53.

Luh, W. M., Wong, E. C., Bandettini, P. A., and Hyde, J. S. (1999). QUIPSS II with thin-slice TIl periodic saturation: a method for improving accuracy of quantitative perfusion imaging using pulsed arterial spin labeling. Magn Reson. Med. 41, 1246-1254.

Mahncke, H.W., Connor, B. B., Appelman, J., Ahsanuddin, O. N., Hardy, J. L., Wood, R. A., Joyce, N. M., Boniske, T., Atkins, S. M., and Merzenich, M. M. (2006). Memory enhancement in healthy older adults using a brain plasticity-based training program: a randomized, controlled study. Proc. Natl. Acad. Sci. U.S.A. 103, 12523-12528.

Marchal, G., Rioux, P., Petit-Taboue, M. C., Sette, G., Travere, J. M., Le Poec,
C., Courtheoux, P., Derlon, J. M., and Baron, J. C. (1992). Regional cerebral oxygen consumption, blood flow, and blood volume in healthy human aging. Arch. Neurol. 49, 1013-1020.

Martin, A. J., Friston, K. J., Colebatch, J. G., and Frackowiak, R. S. (1991). Decreases in regional cerebral blood flow with normal aging. J. Cereb. Blood Flow Metab. 11, 684-689.

May, A., Hajak, G., Ganssbauer, S., Steffens, T., Langguth, B., Kleinjung, T., and Eichhammer, P. (2007). Structural Brain Alterations following 5 Days of Intervention: Dynamic Aspects of Neuroplasticity. Cereb. Cortex 17, 205-210.

Mazoyer, B., Houde, O., Joliot, M., Mellet, E., and Tzourio-Mazoyer, N. (2009). Regional cerebral blood flow increases during wakeful rest following cognitive training. Brain Res. Bull. 80, 133-138.

Meltzer, C. C., Cantwell, M. N., Greer, P. J., Ben-Eliezer, D., Smith, G., Frank, G., Kaye, W. H., Houck, P. R., and Price, J. C. (2000). Does cerebral blood flow decline in healthy aging? A PET study with partial-volume correction. J. Nucl. Med. 41, 1842-1848.

Mora, F., Segovia, G., and del Arco, A. (2007). Aging, plasticity and environmental enrichment: structural changes and neurotransmitter dynamics in several areas of the brain. Brain Res. Rev. 55, 78-88.

Mozolic, J. L., Long, A. B., Morgan, A. R., Rawley-Payne, M., and Laurienti, P. J. (2009). A cognitive training intervention improves modality-specific attention in a randomized controlled trial of healthy older adults. Neurobiol. Aging. [Epub ahead of print].

Nyberg, L., Sandblom, J., Jones, S., Neely, A. S., Petersson, K. M., Ingvar, M., and Backman, L. (2003). Neural correlates of training-related memory improvement in adulthood and aging. Proc. Natl. Acad. Sci. U.S.A. 100, 13728-13733.

Olesen,P.J.,Westerberg, H., and Klingberg, T. (2004). Increased prefrontal and parietal activity after training of working memory. Nat. Neurosci. 7, 75-79.

Park, D. C., Lautenschlager, G., Hedden, T., Davidson, N. S., Smith, A. D., and Smith, P. K. (2002). Models of visuospatial and verbal memory across the adult life span. Psychol. Aging 17 299-320.

Parkes, L. M., Rashid, W., Chard, D. T., and Tofts, P.S. (2004). Normal cerebral perfusion measurements using arterial spin labeling: reproducibility, stability, and age and gender effects. Magn. Reson. Med. 51, 736-743.

Pereira, A. C., Huddleston, D. E., Brickman, A. M., Sosunov, A. A., Hen, R., McKhann, G. M., Sloan, R., 
Gage, F. H., Brown, T. R., and Small, S. A. (2007). An in vivo correlate of exercise-induced neurogenesis in the adult dentate gyrus. Proc. Natl. Acad. Sci. U.S.A. 104, 5638-5643.

Raichle, M.E., MacLeod, A. M., Snyder, A. Z., Powers, W. J., Gusnard, D. A., and Shulman, G. L. (2001). A default mode of brain function. Proc. Natl. Acad. Sci. U.S.A. 98, 676-682.

Ramnani, N., and Owen, A. M. (2004). Anterior prefrontal cortex: insights into function from anatomy and neuroimaging. Nat. Rev. Neurosci. 5, 184-194.

Raz, N., Gunning-Dixon, F., Head, D., Rodrigue, K. M., Williamson, A., and Acker, J. D. (2004). Aging, sexual dimorphism, and hemispheric asymmetry of the cerebral cortex: replicability of regional differences in volume. Neurobiol. Aging 25, 377-396.

Raz, N., Lindenberger, U., Rodrigue, K. M., Kennedy, K. M., Head, D., Williamson, A., Dahle, C., Gerstorf, D., and Acker, J.D. (2005). Regional brain changes in aging healthy adults: general trends, individual differences and modifiers. Cereb. Cortex 15, 1676-1689.

Resnick, S. M., Pham, D. L., Kraut, M. A., Zonderman, A. B., and Davatzikos, C. (2003). Longitudinal magnetic resonance imaging studies of older adults: a shrinking brain. J. Neurosci. 23, 3295-3301.

Reuter-Lorenz, P. A., Jonides, J., Smith, E. E., Hartley, A., Miller, A., Marshuetz, C., and Koeppe, R. A. (2000). Age differences in the frontal lateralization of verbal and spatial working memory revealed by PET. J. Cogn. Neurosci. 12, 174-187.

Rhodes, M. G. (2004). Age-related differences in performance on the
Wisconsin card sorting test: a metaanalytic review. Psychol. Aging 19, 482-494.

Rowe, G., Valderrama, S., Hasher, L., and Lenartowicz, A. (2006). Attentional disregulation: a benefit for implicit memory. Psychol. Aging 21, 826-830.

Rypma, B., Berger, J. S., Genova, H. M., Rebbechi, D., and D'Esposito, M. (2005). Dissociating age-related changes in cognitive strategy and neural efficiency using event-related fMRI. Cortex 41, 582-594.

Salat, D. H., Buckner, R. L., Snyder, A. Z., Greve, D. N., Desikan, R. S., Busa, E., Morris, J. C., Dale, A. M., and Fischl, B. (2004). Thinning of the cerebral cortex in aging. Cereb. Cortex 14, 721-730.

Salat, D. H., Greve, D. N., Pacheco, J. L., Quinn, B. T., Helmer, K. G., Buckner, R. L., and Fischl, B. (2009). Regional white matter volume differences in nondemented aging and Alzheimer's disease. Neuroimage 44, 1247-1258.

Salat, D. H., Tuch, D. S., Hevelone, N. D., Fischl, B., Corkin, S., Rosas, H. D., and Dale, A. M. (2005). Age-related changes in prefrontal white matter measured by diffusion tensor imaging. Ann. N. Y. Acad. Sci. 1064, 37-49.

Shomstein, S., and Yantis, S. (2004). Control of attention shifts between vision and audition in human cortex. J. Neurosci. 24, 10702-10706.

Sohlberg, M.M., and Mateer, C.A. (1987). Effectiveness of an attention-training program. J. Clin. Exp. Neuropsychol. 9, 117-130.

Sohlberg, M. M., McLaughlin, K. A., Pavese, A., Heidrich, A., and Posner, M. I. (2000). Evaluation of attention process training and brain injury education in persons with acquired brain injury. J. Clin. Exp. Neuropsychol. 22, 656-676.

Tellinghuisen, D. J., and Nowak, E. J. (2003). The inability to ignore auditory distractors as a function of visual task perceptual load. Percept. Psychophys. 65, 817-828.

Uludag, K., Dubowitz, D. J., Yoder, E. J., Restom, K., Liu, T. T., and Buxton, R. B. (2004). Coupling of cerebral blood flow and oxygen consumption during physiological activation and deactivation measured with fMRI. Neuroimage 23, 148-155.

Velanova, K., Jacoby, L. L., Wheeler, M. E., McAvoy, M. P., Petersen, S. E., and Buckner, R. L. (2003). Functionalanatomic correlates of sustained and transient processing components engaged during controlled retrieval. J. Neurosci. 23, 8460-8470.

Verhaeghen, P., and Cerella, J. (2002). Aging, executive control, and attention: a review of meta-analyses. Neurosci. Biobehav. Rev. 26, 849-857.

Weissman, D. H., Warner, L. M., and Woldorff, M. G. (2004). The neural mechanisms for minimizing crossmodal distraction. J. Neurosci. 24, 10941-10949.

Willis, S. L., Tennstedt, S. L., Marsiske, M., Ball, K., Elias, J., Koepke, K. M., Morris, J. N., Rebok, G. W., Unverzagt, F. W., Stoddard,A.M., and Wright, E. (2006). Long-term effects of cognitive training on everyday functional outcomes in older adults. JAMA 296, 2805-2814.

Wong, E. C., Buxton, R. B., and Frank, L. R. (1998). Quantitative imaging of perfusion using a single subtraction (QUIPSS and QUIPSS II). Magn. Reson. Med. 39, 702-708.
Yang, L., and Hasher, L. (2007). The enhanced effects of pictorial distraction in older adults. J. Gerontol. B Psychol. Sci. Soc. Sci. 62:P230-233.

Yang, Y. (2002). Perfusion MR imaging with pulsed arterial spin-labeling: basic principles and applications in functional brain imaging. Concepts Magn. Reson. Imaging 14, 347-357.

Yang, Y., Frank, J. A., Hou, L., Ye, F. Q., McLaughlin, A. C., and Duyn, J. H. (1998). Multislice imaging of quantitative cerebral perfusion with pulsed arterial spin labeling. Magn. Reson. Med. 39, 825-832.

Conflict of Interest Statement: Authors Jennifer L. Mozolic and Paul J. Laurienti, and Wake Forest University School of Medicine have a provisional patent for the training program described in this manuscript. Author Satoru Hayasaka has no conflicts of interest to declare.

Received: 18 September 2009; paper pending published:05 November 2009; accepted: 06 February 2010; published online: 12 March 2010.

Citation: Mozolic JL, Hayasaka S, and Laurienti PJ (2010) A cognitive training intervention increases resting cerebral blood flow in healthy older adults. Front. Hum. Neurosci. 4:16. doi: 10.3389/neuro.09.016.2010

Copyright (C) 2010 Mozolic, Hayasaka, and Laurienti. This is an open-access article subject to an exclusive license agreement between the authors and the Frontiers Research Foundation, which permits unrestricted use, distribution, and reproduction in any medium, provided the original authors and source are credited. 